\title{
Estudio de tiempos como base para trazar estrategias orientadas al incremento de la eficiencia del proceso de batido de una planta de producción de helados
}

André Gianfranco Alfaro Pacheco ${ }^{1}$ Rosa Karol MoOre Torres ${ }^{2}$

Recibido: 06/09/2019 Aceptado: 04/12/2019 Publicado: 16/10/2020

\begin{abstract}
RESUMEN
Esta investigación se aplicó al proceso de batido de una planta de producción de helados de la empresa Delihelados, ubicada en Bogotá, Colombia. En ella existe un inadecuado uso del personal en el balanceo de las líneas de batido. Se analizaron los tiempos del proceso de batido de los sabores y presentaciones que representan el $80 \%$ de las ventas, los cuales son Cubetas retornables, Cubeta transparente Perú y Litros, con el fin de hallar los cuellos de botella y establecer estrategias que reduzcan los tiempos. La población a analizar fue el cliente interno, esto es, 13 operarios que laboran en la planta en el turno de la mañana. Los resultados evidencian que el cuello de botella es la actividad de llenado, así como sus limitantes en cuanto a las variables eficiencia $(63 \%, 64 \%$ y $63 \%)$ y número de operarios (3, 3 y 4) para las presentaciones de CR, CTP y Litro (vainilla). Además, se pudo determinar que, balanceando las líneas de las presentaciones analizadas, hay una oportunidad potencial de mejorar la eficiencia y los recursos usados en estas.
\end{abstract}

Palabras clave: estudio de tiempos; tiempo estándar; eficiencia; proceso de batido.

\section{INTRODUCCIÓN}

Actualmente, las empresas están tratando de unificar sus procesos mediante el empleo de normas y estándares, con el propósito de aumentar la ventaja competitiva, la eficiencia y la productividad de estos procesos, y así evitar duplicidades y estrés laboral.

La presente investigación se interesa por el proceso de batido de una planta de producción de helados de la empresa Delihelados, ubicada en Bogotá, Colombia. Allí se observó un uso inadecuado del personal en el balanceo de las líneas de batido, ya que la elaboración se realizaba empíricamente y sin un sustento ingenieril. En este artículo, se reconoce la importancia de proporcionar técnicas de estudio de tiempos que faciliten racionalizar los recursos involucrados en el proceso de batido de helados; de esta manera, se evitan los desperdicios, se disminuyen las horas muertas de los operarios, se genera el aumento de la eficiencia y se reducen los errores, lo que permite conocer a fondo los lineamientos para ejecutar cada actividad de este proceso.

Con respecto al objetivo, este busca analizar los tiempos de las presentaciones más vendidas de la planta de producción de Delihelados, con la finalidad de identificar los cuellos de botella y establecer estrategias que reduzcan los tiempos de procesamiento. Cabe señalar que el estudio se enfrentó a ciertas limitaciones, entre ellas, la espera de la programación de los productos que debían a analizarse. Asimismo, la falta de trabajos previos acerca de los tiempos de la línea de batido en una planta de producción de helados implica un obstáculo, es por ello que se espera lograr una investigación que contribuya en este sentido.

1 Ingeniero de alimentos por la Universidad Nacional San Luis Gonzaga de Ica (Ica, Perú). Actualmente, es consultor independiente. (Lima, Perú). ORCID: https://orcid.org/0000-0002-6401-8854

E-mail: andreee2@hotmail.com

2 Doctora en Administración por la Universidad Nacional Federico Villarreal (Lima, Perú). Actualmente, se desempeña como docente de posgrado en la Facultad de Ingeniería Industrial de la Universidad Nacional Mayor de San Marcos. (Lima, Perú).

ORCID: https://orcid.org/0000-0002-7608-9377

E-mail: kmooret@unmsm.edu.pe 
A modo de soluciones de estos inconvenientes, se proponen algunos trabajos para orientar la estructura de este artículo. Entre ellos está el de Muñoz (2006), quien realiza un estudio acerca de las operaciones de elaboración de helados, con el fin de mejorar los procesos de producción. La mejora se basa en los estándares obtenidos mediante la toma de tiempos y movimientos, la elaboración de las fichas técnicas y los formatos de control de calidad. Por su parte, González y Jácome (2012) presentan una tesis en la que aplicaron herramientas de mejora para aumentar la productividad. Los autores recomiendan adquirir maquinaria para reducir el cuello de botella en 13 y 9 minutos. Igualmente, las ventajas de este tipo de estudio son el seguimiento del desempeño laboral, la reducción del tiempo para la ejecución de tareas y de costos, la comparación de eficiencia de los métodos de trabajo, la conservación de recursos, entre otros.

Finalmente, Alfonso y Tamayo (2016) aplican la metodología Lean Manufacturing en el proceso de batido para una planta de helados para corroborar que, mediante una gestión de indicadores y mantenimiento preventivo de equipos, aumenta la productividad. Los resultados demuestran el incremento de 80 órdenes promedio mes pro técnico a 120 órdenes promedio mes pro técnico (se define como mes pro técnico al número de programaciones producción de mix planeadas en el mes).

\section{METODOLOGÍA}

La investigación es aplicada y explicativa, con enfoque cuantitativo y diseño preexperimental transversal. La población que se analizó fue el cliente interno del turno mañana, representado por 13 operarios del proceso de batido. Se aplicaron técnicas de estudio de tiempos, pero, por criterios de delimitación técnica, no se abordaron aspectos relacionados a la ingeniería de métodos.

El objetivo de emplear esta metodología es buscar la ampliación del conocimiento en el área de procesos de batido de una planta de producción de helados y su mejora, específicamente, en la identificación de cuellos de botella que aparecen en este proceso.

\section{ANÁLISIS}

\section{Procesos analizados}

Para este estudio de tiempos en el proceso de batido, se determinaron los sabores y presentaciones más vendidos en la empresa Delihelados; asimismo, se consideraron otras variables, como el número de operarios, el impacto en el costo, etc. Rodríguez (2008) destaca que decidir el orden de las operaciones a medir depende del objetivo general de la medición. Para ello, se pueden emplear criterios como el orden de las operaciones, tomando en cuenta su presentación dentro del proceso, la posibilidad de ahorro en la operación y el costo anual de operación (Meyers, 2000).

Para establecer los productos que representan el $80 \%$ de las ventas, se aplicó el principio de $\mathrm{Pa}-$ reto en las presentaciones de la línea de Helado Gourmet. Con ello se determinó que aquellas que cumplían ese requisito fueron las Cubetas retornables (CR), Cubeta transparente Perú (CTP) y Litros. Luego, se registraron los datos de tiempos de las presentaciones más vendidas para el sabor vainiIla. En el trabajo, se realizó un estudio de tiempos, balanceo y eficiencia de la línea para las presentaciones mencionadas. Según Chase, Jacobs y Aquilano (2009), la gráfica de Pareto permite el reconocimiento de aquella actividad que está compuesta por factores o variables que favorecen su dinámica, expresándola en la ley $80 / 20$, es decir, el $80 \%$ de los efectos son originados por el $20 \%$ de los factores o variables también Ilamados "los pocos factores vitales" que condicionan su desempeño.

Por otra parte, según Albi (1992), la eficiencia no debe ser considerada en relación con la variable consumo, sino con la variable producción, puesto que se busca evitar el despilfarro en la producción.

Respecto a la técnica de recolección de datos, el estudio de tiempos se realizó utilizando un cronómetro. Según García (2005), existen diversos tipos de técnicas empleadas para definir un estándar, las cuales se moldean para diferentes usos y se acomodan a diferentes exactitudes y costos.

\subsection{Estudio de tiempos para CR sabor vainilla}

El proceso de batido de CR involucró 3 operarios y 6 actividades, las cuales son: llenado; tapado y colocado de termoencogible; apilado de CR; termosellado; codificado y colocado en canastilla, y traslado de ida y regreso. Se realizó la medición de tiempos para el sabor vainilla, para lo cual, se colocó a operarios calificados en la línea de batido, ya que sus eficiencias individuales eran similares. Kanawaty (1996) señala que, para aplicar el método estadístico, se necesita efectuar cierto número de observaciones preliminares (n) para utilizar la fórmula del tamaño de la muestra.

En las hojas de trabajo adjuntas, se observan distintos elementos que se explicarán a continuación. 
De acuerdo con Salazar (26 de junio de 2019), el factor de valoración es el porcentaje de calificación global en razón del ritmo estándar para todos los operarios en conjunto; esta ponderación es muy relativa, ya que dicho porcentaje es determinado a criterio del metodista. Las unidades que se sujetan representan el número de unidades de medida en que un operario sujeta un número determinado de productos. El $n$ es el tamaño de la muestra adicional. Heizer y Render (2009) manifiestan que el tamaño de la muestra es importante en la credibilidad de la representatividad de la muestra y que el número de observaciones se basa en estos tres aspectos: la precisión, el nivel de confianza deseado y la variación en los elementos de las tareas. El tiempo observado es el promedio de los tiempos registrados, es decir, "el tiempo que se tarda en ejecutar una tarea o combinación de tareas según lo indica una medición directa" (Caso, 2006, p. 43). El tiempo normal es la multiplicación de tiempo observada por el factor de valoración. Al respecto, Hodson (2009) califica el tiempo normal como el tiempo necesario que toman los operarios en la realización de una labor mediante un método establecido y a un ritmo normal. La casilla suplemento refiere al suplemento de tolerancias que se le da a la fuerza de trabajo (tolerancia: $100+$ porcentaje de suplementos). Cruelles (2013) recomienda la tabla de suplementos de descanso proporcionada por la OIT. El tiempo estándar, siguiendo a Meyers y Stephens (2006), es el tiempo que se necesita para la elaboración de un producto en una fábrica manufacturera, el cual debe contar con las siguientes condiciones: operarios calificados y capacitados, manufactura a ritmo normal y una tarea específica.

En la Tabla 1, se puede determinar que la actividad cuello de botella en el batido de la presentación CR sabor vainilla es el llenado, que tiene un tiempo estándar de 26.80 s/unidad.

En las tablas 2 y 3 , se observa que la eficiencia de línea es de $63 \%$ con 3 operarios trabajando en la línea, pero el análisis de balanceo de línea señala

Tabla 1. Hoja de trabajo de estudio de tiempos para la presentación CR sabor vainilla.

\begin{tabular}{|c|l|c|c|c|c|c|c|}
\hline $\begin{array}{c}\mathbf{N} .{ }^{\circ} \text { de } \\
\text { actividad }\end{array}$ & Actividad & $\begin{array}{c}\mathbf{V} \\
\text { (factor de } \\
\text { valoración } \\
\text { del ritmo de } \\
\text { trabajo) }\end{array}$ & $\begin{array}{c}\text { Unidades que se } \\
\text { sujetan o } \mathbf{~}^{\circ} \text { de } \\
\text { unidades de medi- } \\
\text { da en que se toma } \\
\text { el producto }\end{array}$ & $\begin{array}{c}\text { Tiempo } \\
\text { observado } \\
(\mathbf{s})\end{array}$ & $\begin{array}{c}\text { Tiempo } \\
\text { básico o } \\
\text { normal } \\
\mathbf{( s )}\end{array}$ & $\begin{array}{c}\text { Suplemento } \\
\text { (\%) }\end{array}$ & $\begin{array}{c}\text { Tiempo } \\
\text { estándar o } \\
\text { tiempo tipo } \\
\text { (s) }\end{array}$ \\
\hline 1 & Llenado & 1 & 1 & 26.80 & 26.80 & 1.00 & 26.80 \\
\hline 2 & $\begin{array}{l}\text { Tapado y colocado de } \\
\text { termoencogible }\end{array}$ & 1.02 & 1 & 7.26 & 7.40 & 1.07 & 7.92 \\
\hline 3 & Apilado de CR & 1.02 & 8 & 15.55 & 15.24 & 1.05 & 16.00 \\
\hline 4 & Termosellado & 0.98 & 1 & 4.71 & 4.81 & 1.03 & 4.95 \\
\hline 5 & $\begin{array}{l}\text { Codificado y colocado en } \\
\text { canastilla }\end{array}$ & 0.96 & 3 & 4.94 & 4.84 & 1.03 & 4.99 \\
\hline 6 & Traslado de ida y regreso & & 10.53 & 10.11 & 1.06 & 10.72 \\
\hline
\end{tabular}

Fuente: Elaboración propia.

Tabla 2. Análisis de balanceo de línea para la presentación CR sabor vainilla.

\begin{tabular}{|c|c|c|c|c|c|c|c|c|}
\hline $\begin{array}{l}\mathrm{N} \cdot{ }^{\circ} \text { de } \\
\text { actividad }\end{array}$ & Actividad & $\begin{array}{c}\text { Tiempo } \\
\text { estándar } \\
\text { (s/unidad) }\end{array}$ & $\begin{array}{l}\text { Tiempo } \\
\text { estándar } \\
\text { (s/3 uds.) }\end{array}$ & $\begin{array}{l}\text { Canastillas } \\
\text { * operario/ } \\
\text { min }\end{array}$ & $\begin{array}{c}\mathrm{N} .{ }^{\circ} \text { de } \\
\text { operarios }\end{array}$ & $\begin{array}{c}\text { Unidades } \\
\text { totales } \\
\text { (n. } .^{\circ} \text { de canas- } \\
\text { tillas } / \mathrm{min} \text { ) }\end{array}$ & $\begin{array}{c}\text { Tiempo que } \\
\text { se tarda } 1 \\
\text { operario en } \\
\text { producir } 1 \\
\text { unidad } \\
\end{array}$ & $\begin{array}{c}\text { Tiempo } \\
\text { máximo } \\
\text { usado para } \\
\text { producir } 1 \\
\text { unidad } \\
\end{array}$ \\
\hline 1 & Llenado & 26.77 & 80.31 & 0.75 & 1 & 0.75 & 26.77 & 26.77 \\
\hline 2 & $\begin{array}{l}\text { Tapado y colocado de } \\
\text { termoencogible }\end{array}$ & 7.92 & 23.76 & 2.53 & 1 & 2.53 & 7.92 & 26.77 \\
\hline 3 & Apilado de CR & 16 & 6 & 10 & & 10 & 2 & \\
\hline 4 & Termosellado & 4.95 & 14.86 & 4.04 & & 4.04 & 4.95 & \\
\hline 5 & $\begin{array}{l}\text { Codificado y colocado en } \\
\text { canastilla }\end{array}$ & 4.99 & 14.96 & 4.01 & 1 & 4.01 & 4.99 & 26.77 \\
\hline 6 & Traslado de ida y regreso & 10.72 & 10.72 & 5.6 & & 5.6 & 3.57 & \\
\hline & & & & & 3 & & 50.2 & 80.31 \\
\hline
\end{tabular}

Fuente: Elaboración propia. 
que la eficiencia podría aumentar a un $94 \%$ si se emplean 2 operarios.

\subsection{Estudio de tiempos para CTP sabor vainilla}

El proceso de batido de CTP involucró 3 operarios y 5 actividades, las cuales son: Ilenado, tapado, apilado de CTP, colocado de CTP en canastilla y traslado de ida y regreso.
En la Tabla 4, se determina que la actividad cuello de botella en el batido de la presentación CTP sabor vainilla es el llenado, con un tiempo estándar de 21.02 s/unidad.

En las tablas 5 y 6 , se evidencia que la eficiencia de línea es de $64 \%$ con 3 operarios trabajando en la línea, pero el análisis de balanceo de línea indica

Tabla 3. Resumen del balanceo de línea para la presentación CR sabor vainilla.

\begin{tabular}{|l|c|c|c|c|}
\hline Cuello de botella & 0.75 & canastillas/min & & \\
\hline Rendimiento por hora & 44.82 & canastillas/h & & \\
\hline Duración del proceso & 6.51 & $\mathrm{~h}$ & 23424.5455 & $\mathrm{~s}$ \\
\hline N. ${ }^{\circ}$ de operarios & 3 & & & \\
\hline Productividad & 14.94 & canastillas/h * operario & & \\
\hline Eficiencia de línea & 63 & $\%$ & & \\
\hline Costo horas hombre & 81.32 & miles de pesos & & \\
\hline Tiempo de ciclo & 26.77 & s/unidad & & \\
\hline N. ${ }^{\circ}$ de estaciones recomendadas & 2.00 & & & \\
\hline Tiempo de ciclo & 26.8 & s/unidad & & \\
\hline N. ${ }^{\circ}$ de estaciones recomendadas & 2 & & & \\
\hline Eficiencia potencial & 94 & $\%$ & & \\
\hline
\end{tabular}

Fuente: Elaboración propia.

Tabla 4. Hoja de trabajo de estudio de tiempos para la presentación CTP sabor vainilla.

\begin{tabular}{|c|l|c|c|c|c|c|c|}
\hline $\begin{array}{c}\mathbf{N} .{ }^{\circ} \text { de } \\
\text { actividad }\end{array}$ & \multicolumn{1}{|c|}{ Actividad } & $\begin{array}{c}\mathbf{V} \\
\text { (factor de } \\
\text { valoración } \\
\text { del ritmo de } \\
\text { trabajo) }\end{array}$ & $\begin{array}{c}\text { Unidades que se } \\
\text { sujetan o n. } .^{\circ} \text { de } \\
\text { unidades de medida } \\
\text { en que se toma el } \\
\text { producto }\end{array}$ & $\begin{array}{c}\text { Tiempo } \\
\text { observado } \\
\text { (s) }\end{array}$ & $\begin{array}{c}\text { Tiempo } \\
\text { básico o } \\
\text { normal } \\
(\mathbf{s})\end{array}$ & $\begin{array}{c}\text { Suplemento } \\
\%\end{array}$ & $\begin{array}{c}\text { Tiempo } \\
\text { estándar o } \\
\text { tiempo tipo } \\
\text { (s) }\end{array}$ \\
\hline 1 & Llenado & 1 & 1 & 21.02 & 21.02 & 1.00 & 21.02 \\
\hline 2 & Tapado & 1.02 & 1 & 3.95 & 4.03 & 1.07 & 4.31 \\
\hline 3 & Apilado de CTP & 0.98 & 9 & 49.47 & 48.48 & 1.05 & 5.66 \\
\hline 4 & Colocado en canastilla & 0.98 & 1 & 4.82 & 4.72 & 1.03 & 4.86 \\
\hline 5 & Traslado de ida y regreso & 0.96 & 3 & 10.53 & 10.11 & 1.06 & 10.72 \\
\hline
\end{tabular}

Fuente: Elaboración propia.

Tabla 5. Análisis de balanceo de línea para la presentación CTP sabor vainilla.

\begin{tabular}{|c|l|c|c|c|c|c|c|c|}
\hline $\begin{array}{c}\mathbf{N} .{ }^{\circ} \text { de } \\
\text { actividad }\end{array}$ & Actividad & $\begin{array}{c}\text { Tiempo } \\
\text { estándar } \\
\text { (s/unidad) }\end{array}$ & $\begin{array}{c}\text { Tiempo } \\
\text { estándar } \\
\text { (s/3 uds.) }\end{array}$ & $\begin{array}{c}\text { Canastillas * } \\
\text { operario/min }\end{array}$ & $\begin{array}{c}\text { N. }{ }^{\circ} \text { de } \\
\text { operarios }\end{array}$ & $\begin{array}{c}\text { Unidades } \\
\text { totales } \\
\text { (n. } \text { de }^{\text {demin) }} \\
\text { canastillas/min) }\end{array}$ & $\begin{array}{c}\text { Tiempo que } \\
\text { se tarda 1 } \\
\text { operario en } \\
\text { producir 1 } \\
\text { unidad }\end{array}$ & $\begin{array}{c}\text { Tiempo } \\
\text { máximo } \\
\text { usado para } \\
\text { producir 1 } \\
\text { unidad }\end{array}$ \\
\hline 1 & Llenado & 21.02 & 63.07 & 0.95 & 1 & 0.95 & 21.02 & 21.02 \\
\hline 2 & Tapado & 4.31 & 12.94 & 4.64 & 1 & 4.64 & 4.31 & 21.02 \\
\hline 3 & Apilado de CTP & 50.90 & 16.97 & 3.54 & 1 & 3.54 & 6.36 & 21.02 \\
\hline 4 & $\begin{array}{l}\text { Colocado en } \\
\text { canastilla }\end{array}$ & 4.86 & 14.59 & 4.11 & & 4.11 & 4.86 & \\
\hline 5 & $\begin{array}{l}\text { Traslado de ida y } \\
\text { regreso }\end{array}$ & 10.72 & 10.72 & 5.60 & & 5.60 & 3.57 & \\
\hline & & & & & 3 & & 40.13 & 63.07 \\
\hline
\end{tabular}

Fuente: Elaboración propia. 
que la eficiencia podría aumentar a un $95 \%$ si intervienen 2 operarios.

\subsection{Estudio de tiempos para Litro sabor vainilla}

El proceso de batido de Litros involucró 4 operarios y 6 actividades, las cuales son: llenado, tapado, colocado de termoencogible, termosellado, codificado, y colocado en canastilla y puesta en banda transportadora. En la Tabla 7, se muestra el estudio de tiempos de la presentación Litro sabor vainilla.

En la Tabla 8, se determinó que la actividad cuello de botella en el batido de la presentación Litro sabor vainilla es el llenado, que presenta un tiempo estándar de 5.42 s/unidad (litro). En la Tabla 9, se observa que la eficiencia de línea es de $63 \%$ con 4 operarios en la línea, pero el análisis de balanceo de línea indica que la eficiencia podría aumentar al $84 \%$ si se emplea 3 operarios.

\section{Propuesta de estrategias para mejorar la efi- ciencia del batido}

Mediante los datos obtenidos, se pueden proponer las estrategias que se expondrán a continuación, para mejorar la eficiencia del batido. En cuanto a la actividad de llenado para las presentaciones CR, CTP y Litro, se puede reducir su tiempo con la adquisición de una batidora más eficiente o repotenciando la capacidad de congelado del helado, lo que aumentaría, a su vez, la velocidad de salida. Asimismo, se recomienda balancear los sabores en las presentaciones estudiadas, que representan el $80 \%$ de las ventas en Delihelados, con el fin de tener un impacto en su eficiencia.

De otra parte, también se debería unir dos máquinas batidoras de helado que tengan la misma capacidad, las cuales, por medio de una sola tubería, podrán reducir la salida del llenado a la mitad del

Tabla 6. Resumen del balanceo de línea para la presentación CTP sabor vainilla.

\begin{tabular}{|l|c|c|c|c|}
\hline Cuello de botella & 0.95 & canastillas/min & & \\
\hline Rendimiento por hora & 57.08 & canastillas/h & & \\
\hline Duración del proceso & 5.84 & $\mathrm{~h}$ & 21021.82 & $\mathrm{~s}$ \\
\hline $\mathrm{N} .{ }^{\circ}$ de operarios & 3 & & & \\
\hline Productividad & 19.03 & $\begin{array}{c}\text { canastillas/h } \\
\text { operario }\end{array}$ & & \\
\hline Eficiencia de línea & 64 & \% & & \\
\hline Costo horas hombre & 72.98 & miles de pesos & & \\
\hline Tiempo de ciclo & 21.02 & s/unidad & & \\
\hline $\mathrm{N} .{ }^{\circ}$ de estaciones recomendadas & 2 & & & \\
\hline Tiempo de ciclo & 21.02 & s/unidad & & \\
\hline $\mathrm{N} .{ }^{\circ}$ de estaciones recomendadas & 2 & & & \\
\hline Eficiencia potencial & 95 & $\%$ & & \\
\hline
\end{tabular}

Fuente: Elaboración propia.

Tabla 7. Hoja de trabajo de estudio de tiempos para la presentación Litros sabor vainilla.

\begin{tabular}{|c|l|c|c|c|c|c|c|}
\hline $\begin{array}{c}\mathbf{N} .{ }^{\circ} \mathbf{d e} \\
\text { actividad }\end{array}$ & Actividad & $\begin{array}{c}\mathbf{V} \\
\text { (factor de } \\
\text { valoración } \\
\text { del ritmo de } \\
\text { trabajo) }\end{array}$ & $\begin{array}{c}\text { Unidades que se } \\
\text { sujetan o } .^{\circ} \text { de } \\
\text { unidades de medida } \\
\text { en que se toma el } \\
\text { producto }\end{array}$ & $\begin{array}{c}\text { Tiempo } \\
\text { observado } \\
\mathbf{( s )}\end{array}$ & $\begin{array}{c}\text { Tiempo } \\
\text { básico o } \\
\text { normal } \\
(\mathbf{s})\end{array}$ & $\begin{array}{c}\text { Suplemento } \\
\%\end{array}$ & $\begin{array}{c}\text { Tiempo } \\
\text { estándar o } \\
\text { tiempo tipo } \\
\text { (s) }\end{array}$ \\
\hline 1 & Llenado & 1 & 1 & 5.42 & 5.42 & 1.00 & 5.42 \\
\hline 2 & Tapado & 1.04 & 1 & 2.23 & 2.32 & 1.07 & 2.48 \\
\hline 3 & Colocado de termoencogible & 1.02 & 1 & 1.29 & 1.31 & 1.05 & 1.38 \\
\hline 4 & Termosellado & 1.02 & 1 & 1.86 & 1.90 & 1.03 & 1.95 \\
\hline 5 & Codificado & 0.98 & 1 & 0.80 & 0.78 & 1.03 & 0.80 \\
\hline 6 & $\begin{array}{l}\text { Colocado en canastilla y } \\
\text { puesta en banda } \\
\text { transportadora }\end{array}$ & 0.97 & 15 & 23.54 & 22.83 & 1.06 & 24.20 \\
\hline
\end{tabular}

Fuente: Elaboración propia. 
Tabla 8. Análisis de balanceo de línea para la presentación Litro sabor vainilla.

\begin{tabular}{|c|c|c|c|c|c|c|c|c|}
\hline $\begin{array}{l}\mathrm{N} .{ }^{\circ} \text { de } \\
\text { actividad }\end{array}$ & Actividad & $\begin{array}{c}\text { Tiempo } \\
\text { estándar } \\
\text { (s/unidad) }\end{array}$ & $\begin{array}{l}\text { Tiempo } \\
\text { estándar } \\
\text { (s/15 } \\
\text { uds.) }\end{array}$ & $\begin{array}{l}\text { Canastillas * } \\
\text { operario/min }\end{array}$ & $\begin{array}{c}\mathrm{N} .^{\circ} \text { de } \\
\text { operarios }\end{array}$ & $\begin{array}{l}\text { Unidades } \\
\text { totales } \\
\text { ( }\left(.^{\circ} \text { de }\right. \\
\text { canastillas } / \\
\text { min) }\end{array}$ & $\begin{array}{c}\text { Tiempo que } \\
\text { se tarda } 1 \\
\text { operario en } \\
\text { producir } 1 \\
\text { unidad }\end{array}$ & $\begin{array}{l}\text { Tiempo } \\
\text { máximo } \\
\text { usado para } \\
\text { producir } 1 \\
\text { unidad }\end{array}$ \\
\hline 1 & Llenado & 5.42 & 81.37 & 0.74 & 1 & 0.74 & 5.42 & 5.42 \\
\hline 2 & Tapado & 2.48 & 37.17 & 1.61 & 1 & 1.61 & 2.48 & 5.42 \\
\hline 3 & $\begin{array}{l}\text { Colocado de } \\
\text { termoencogible }\end{array}$ & 1.38 & 20.69 & 2.90 & & 2.90 & 1.38 & \\
\hline 4 & Termosellado & 1.95 & 29.30 & 2.05 & 1 & 2.05 & 1.95 & 5.42 \\
\hline 5 & Codificado & 0.80 & 12.06 & 4.98 & 1 & 4.98 & 0.80 & 5.42 \\
\hline \multirow[t]{2}{*}{6} & $\begin{array}{l}\text { Colocado en } \\
\text { canastilla y puesta } \\
\text { en banda } \\
\text { transportadora } \\
\end{array}$ & 24.20 & 24.20 & 2.48 & & 2.48 & 1.61 & \\
\hline & & & & & 4 & & 13.65 & 21.70 \\
\hline
\end{tabular}

Fuente: Elaboración propia.

Tabla 9. Resumen del balanceo de línea para la presentación Litro sabor vainilla.

\begin{tabular}{|l|c|c|c|c|}
\hline Cuello de botella & 0.74 & canastillas/min & & \\
\hline Rendimiento por hora & 44.24 & canastillas/h & & \\
\hline Duración del proceso & 5.65 & $\mathrm{~h}$ & 20342.308 & \\
\hline $\mathrm{N} .{ }^{\circ}$ de operarios & 4 & & & $\mathrm{~s}$ \\
\hline Productividad & 11.06 & canastillas/h * operario & & \\
\hline Eficiencia de línea & 63 & \% & & \\
\hline Costo horas hombre & 94.16 & miles de pesos & & \\
\hline Tiempo de ciclo & 5.42 & s/unidad & & \\
\hline N. ${ }^{\circ}$ de estaciones recomendadas & 3 & & & \\
\hline Tiempo de ciclo & 5.42 & s/unidad (litro) & & \\
\hline N. ${ }^{\circ}$ de estaciones recomendadas & 3 & & & \\
\hline Eficiencia potencial & 84 & $\%$ & & \\
\hline
\end{tabular}

Fuente: Elaboración propia.

tiempo, empleando casi la misma cantidad de operarios, lo que traería consigo una mejora en la eficiencia. Finalmente, se sugiere redistribuir los equipos complementarios y utensilios para el proceso de batido mediante un estudio de métodos, con el fin de reducir el número de operarios y aumentar la eficiencia de la línea.

\section{CONCLUSIONES}

El tiempo estándar para las presentaciones de CR, CTP y Litro para el sabor vainilla fue de $50.2 \mathrm{~s} / \mathrm{CR}$, 40.1 s/CTP y 13.7 s/Litro. En esta distribución, el cuello de botella para todas las presentaciones analizadas fue encontrada en la actividad de llenado.

La eficiencia actual para las presentaciones de CR, CTP y Litro (vainilla) fue de $63 \%$, 64\% y $63 \%$, respectivamente, y la eficiencia potencial para el balanceo de líneas para estas presentaciones sería de $94 \%$, 95\% y $84 \%$, respectivamente.

El número de operarios que actualmente se emplea para batir las presentaciones de CR, CTP y Litro (vainilla) es de 3, 3 y 4, respectivamente, y el número de operarios propuestos a emplear balanceando las líneas de producción sería de 2, 2 y 3, respectivamente.

\section{REFERENCIAS BIBLIOGRÁFICAS}

[1] Albi, E. (1992). Evaluación de la eficiencia pública. (El control de eficiencia del sector público). Hacienda Pública Española, 120-121, 299-316. 
[2] Alfonso, A. y Tamayo, Á. F. (2016). Diseño del sistema de gestión de procesos y operaciones para la administración del mantenimiento industrial en Helados Popsy. (Tesis de maestría). Universidad Sergio Arboleda, Bogotá.

[3] Caso, A. (2006). Sistema de incentivos a la producción. Madrid, España: FC Editorial.

[4] Chase R., Jacobs R. y Aquilano N. (2009). Administración de operaciones. Producción y cadena de suministros. México D. F., México: Mc Graw Hill.

[5] Cruelles, J. A. (2013). Productividad e incentivos. Cómo hacer que los tiempos de fabricación se cumplan. México D. F., México: Alfaomega / Marcombo.

[6] García, R. (2005). Estudio del trabajo. Ingeniería de métodos y medición del trabajo. México D. F., México: McGraw-Hill.

[7] González, L. A. y Jácome, A. H. (2012). Elaboración de una propuesta de mejora para el proceso productivo del helado de crema de una empresa manufacturera en la ciudad de Guayaquil. (Tesis de pregrado). Escuela Superior Politécnica del Litoral, Guayaquil.

[8] Heizer, J. y Render, B. (2009). Principios de administración de operaciones. México D. F., México: Pearson Educación.

[9] Hodson, W. K. (2009). Maynard. Manual del Ingeniero Industrial. México D. F., México: McGraw-Hill.
[10] Kanawaty, G. (1996). Introducción al estudio del trabajo. Ginebra, Suiza: Oficina Internacional del Trabajo.

[11] Meyers, F. E. (2000). Estudios de tiempos y movimientos. Para la manufactura ágil. México D. F., México: Pearson Educación

[12] Meyers, F. E. y Stephens, M. P. (2006). Diseño de instalaciones de manufactura y manejo de materiales. México D. F., México: Pearson Educación.

[13] Muñoz, D. J. (2006). Estandarización de los procesos de producción de los productos elaborados para los puntos de venta de Yogen Früz. (Tesis de pregrado). Universidad de La Salle, Bogotá.

[14] Rodríguez, J. (2008). Determinación del tiempo estándar para la actualización de las ayudas visuales en una línea de producción de una empresa manufacturera. (Tesis de pregrado). Instituto Tecnológico de Sonora, Sonora.

[15] Salazar, B. (26 de junio de 2019). Valoración del ritmo de trabajo. [Entrada de blog]. Ingeniería Online. Recuperado de https://www. ingenieriaindustrialonline.com/estudio-detiempos/valoracion-del-ritmo-de-trabajo. 


\section{Time Study as a Basis to Develop Strategies Aimed at Increasing the Efficiency of the Whipping Process of an Ice Cream Production Plant}

\begin{abstract}
The subject of this research is the whipping process of an ice cream production plant of Delihelados, a company located in Bogotá, Colombia, where an inadequate distribution of personnel is observed on the production lines. Whipping times of the ice cream flavors and product offerings that represent $80 \%$ of sales, which are Cubetas retornables (returnable buckets, CR), Cubetas transparentes Perú (Peru transparent buckets, CTP) and Litro (liter, L), were analyzed in order to identify bottlenecks and develop strategies to reduce production times. The population analyzed was the internal client represented by 13 morning-shift operators. Results show that the bottleneck activity is filling, which depends on line efficiency $(63 \%, 64 \%$ and $63 \%)$ and number of operators ( 3,3 and 4$)$ for CR, CTP and $L$ vanilla product offerings. Furthermore, it was determined that balancing the production lines of the studied product offerings would potentially improve its efficiency and use of resources.
\end{abstract}

Keywords: time study; standard time; efficiency; whipping process.

\section{INTRODUCTION}

Currently, companies are trying to unify their processes through the use of norms and standards, in order to increase the competitive advantage, efficiency and productivity of these processes, and thus avoid redundancy and work-related stress.

This research is focused on the whipping process of an ice cream production plant of Delihelados, a company located in Bogotá, Colombia. An inadequate line balancing in the whipping process was observed, as ice cream production was conducted empirically and without an engineering basis. In this article, the importance of providing time study techniques that enable the rationalization of the resources involved in the ice cream whipping process is recognized. Thus, waste is avoided, operators' idle hours are reduced, efficiency levels are increased and errors are reduced, making it possible to gain a thorough understanding of the procedures to perform each activity of this process.

This research aims at analyzing production times of the best-selling products from the Delihelados production plant, in order to identify bottlenecks and establish strategies that reduce production times. It should be noted that the study encountered certain shortcomings, including the delay in scheduling the whipping of the products to be analyzed. Also, the lack of previous studies on production times regarding the whipping process in this production plant represents an obstacle; therefore, this research is expected to contribute in that sense.

As solutions to these problems, some studies are proposed to provide guidance in the structuring of this article. Among them is that of Muñoz (2006), who conducts a study about ice cream manufacturing operations, in order to improve production processes. The improvement is based on the standards obtained through

1 Food engineer from the Universidad Nacional San Luis Gonzaga de Ica (Ica, Peru). Currently working as an independent consultant. (Lima, Peru) ORCID: https://orcid.org/0000-0002-6401-8854

E-mail: andreee2@hotmail.com

$2 \mathrm{PhD}$ in Administration from the Universidad Nacional Federico Villarreal (Lima, Perú). Currently working as postgraduate professor at the School of Industrial Engineering of the Universidad Nacional Mayor de San Marcos. (Lima, Peru).

ORCID: https://orcid.org/0000-0002-7608-9377

E-mail: kmooret@unmsm.edu.pe 
the study of time and motion, technical data sheets and quality control formats. For their part, González and Jácome (2012) present a thesis in which they applied improvement tools to increase productivity. The authors recommend purchasing machinery to reduce the bottleneck wait times for packaging and sealing processes by 13 and 9 minutes, respectively. Likewise, the advantages of this type of study are work performance monitoring, costs reduction, time reduction for the execution of tasks, comparison of working methods efficiency, saving of resources, among others.

Finally, Alfonso and Tamayo (2016) apply the Lean Manufacturing methodology in the whipping process of an ice cream plant to corroborate that, through the management of indicators and equipment preventive maintenance, it increases productivity. The results demonstrate the increase from an average of 80 to 120 orders per mes pro técnico (scheduled mix production per month).

\section{METHODOLOGY}

This is an applied, explanatory research with a quantitative approach and cross-sectional, pre-experimental design. The population analyzed was the internal client represented by 13 operators of the whipping process working the morning shift. Time study techniques were applied; however, by technical delimitation criteria, no aspects related to method engineering were addressed.

The objective of employing this methodology is to broaden knowledge about the whipping process of an ice cream production plant and its improvement, specifically, the identification of bottlenecks that are encountered in this process.

\section{ANALYSIS}

\section{Analyzed processes}

For the time study in the whipping process, the best-selling Delihelados flavors and product offerings were determined; other variables, such as the number of operators, the impact on the cost, etc., were also considered. Rodríguez (2008) notes that determining the order of operations to be measured depends on the general purpose of the measurement. For that, criteria such as the order of operations as presented in the process, the possibility of time saving and the annual cost of operation can be used (Meyers, 2000).

To determine the products that account for $80 \%$ of sales, the Pareto principle was applied to the Helado
Gourmet line product offerings. Thus, it was determined that those products were the Cubetas retornables (returnable buckets, CR), Cubetas transparentes Perú (Peru transparent buckets, CTP) and Litro (liter, L). Then, time data of the best-selling vanilla product offerings was recorded. Time study, line balancing and line efficiency of the abovementioned product offerings were studied in this research. According to Chase, Jacobs and Aquilano (2009), the Pareto chart makes it possible to recognize that activity composed of factors or variables that favor its dynamics, expressing it in the $80 / 20$ rule, that is, $80 \%$ of outcomes result from $20 \%$ of the factors or variables, also called the "vital few" factors that condition their performance.

On the other hand, according to Albi (1992), efficiency should not be related to the consumption variable, but rather to the production variable, as the aim is to avoid waste in production.

Regarding the data collection technique, the time study was performed using a stopwatch. According to García (2005), there are several types of techniques used to define a standard, which are adapted for different uses, specifications and costs.

\subsection{Time study for the vanilla $C R$ product offering}

The CR whipping process involved 3 operators and 6 activities, which are: filling, capping and placing of heat shrink film, stacking, thermosealing, coding and placement in basket, and transport of basket to conveyor belt and back. Time measurement for the vanilla flavor was conducted, for which, qualified operators were placed in the whipping line as their individual efficiency levels were similar. Kanawaty (1996) states that, in order to apply the statistical method, a number of preliminary observations ( $n$ ) are required to use the sample size formula.

In the attached record sheets, various elements are noted and will be explained below. According to Salazar (June 26, 2019), the rating factor is the percentage of overall performance rate based on the standard performance rate of operators as a whole; this value is very relative, given that this percentage is determined at the time study expert's discretion. The units to be hold represent the number of products an operator holds during a determined period. $\mathrm{N}$ represents the size of the additional sample. Heizer and Render (2009) state that the size of the sample is important in the credibility of the representativeness of the sample and that the number of observations is based on these three aspects: accuracy, desired level of confidence and variation within the tasks elements. Observed time is the average of recorded 
times, i.e., "el tiempo que se tarda en ejecutar una tarea o combinación de tareas según lo indica una medición directa" [the time it takes to execute a task or combination of tasks as indicated by a direct measurement] (Case, 2006, p. 43), whereas normal time is the multiplication of observed time by performance rating factor. In this regard, Hodson (2009) describes normal time as the time taken by operators to perform a task using an established method at a normal pace. The allowance box refers to the time provided to the workers (tolerance: $100+$ allowance percent). Cruelles (2013) recommends the table of rest allowances provided by the ILO. The standard time, following Meyers and Stephens (2006), is the time needed for the production of a product in a manufacturing factory, which should meet the following conditions: skilled and trained workers, manufacturing at a normal pace and a specific task.
In Table 1, it is determined that the bottleneck activity for the vanilla CR product offering is filling, with a standard time of 26.80 s/unit.

In Tables 2 and 3, a line efficiency of $63 \%$ is observed with 3 operators on the line; however, line balancing analysis indicates that efficiency could increase to $94 \%$ if 2 operators were employed.

\subsection{Time study for vanilla CTP product offering}

The CTP whipping process involved 3 operators and 5 activities, which are: filling, capping, CTP stacking, placement of CTP in a basket, and transport of basket to conveyor belt and back.

In Table 4, it is determined that the bottleneck activity in vanilla CTP product offering is filling, with a standard time of 21.02 s/unit.

In Tables 5 and 6 , a line efficiency of $64 \%$ is observed with 3 operators on the line; however, line

Table 1. Time study record sheet for vanilla CR product offering.

\begin{tabular}{|c|l|c|c|c|c|c|c|}
\hline $\begin{array}{c}\text { Order of } \\
\text { activity }\end{array}$ & Activity & $\begin{array}{c}\mathbf{V} \\
\text { (performance } \\
\text { rating factor) }\end{array}$ & $\begin{array}{c}\text { Number of units } \\
\text { hold during certain } \\
\text { period }\end{array}$ & $\begin{array}{c}\text { Observed } \\
\text { time } \\
\mathbf{( s )}\end{array}$ & $\begin{array}{c}\text { Basic or } \\
\text { Normal } \\
\text { time } \\
(\mathbf{s})\end{array}$ & $\begin{array}{c}\text { Allowance } \\
\text { time } \\
(\%)\end{array}$ & $\begin{array}{c}\text { Standard } \\
\text { time } \\
(\mathbf{s})\end{array}$ \\
\hline 1 & Filling & 1 & 1 & 26.80 & 26.80 & 1.00 & 26.80 \\
\hline 2 & $\begin{array}{l}\text { Capping and placing of } \\
\text { heat shrink film }\end{array}$ & 1.02 & 1 & 7.26 & 7.40 & 1.07 & 7.92 \\
\hline 3 & CR stacking & 0.98 & 8 & 15.55 & 15.24 & 1.05 & 16.00 \\
\hline 4 & Thermosealing, & 1.02 & 1 & 4.71 & 4.81 & 1.03 & 4.95 \\
\hline 5 & $\begin{array}{l}\text { Coding and placement in } \\
\text { basket }\end{array}$ & 0.98 & 1 & 4.94 & 4.84 & 1.03 & 4.99 \\
\hline 6 & $\begin{array}{l}\text { Transport of basket to } \\
\text { conveyor belt and back }\end{array}$ & 0.96 & 3 & 10.53 & 10.11 & 1.06 & 10.72 \\
\hline
\end{tabular}

Source: Prepared by the authors.

Table 2. Line balancing analysis for vanilla CR product offering.

\begin{tabular}{|c|l|c|c|c|c|c|c|c|}
\hline $\begin{array}{c}\text { Order of } \\
\text { activity }\end{array}$ & Activity & $\begin{array}{c}\text { Standard } \\
\text { time } \\
\text { (s/unit) }\end{array}$ & $\begin{array}{c}\text { Standard } \\
\text { time } \\
\text { (s/3 units) }\end{array}$ & $\begin{array}{c}\text { Baskets * } \\
\text { operator/ } \\
\text { min }\end{array}$ & $\begin{array}{c}\text { No. of } \\
\text { operators }\end{array}$ & $\begin{array}{c}\text { Total units } \\
\text { (No. of bas- } \\
\text { kets/min) }\end{array}$ & $\begin{array}{c}\text { Time it takes } \\
\text { an operator } \\
\text { to produce a } \\
\text { unit }\end{array}$ & $\begin{array}{c}\text { Maximum } \\
\text { time used } \\
\text { to produce } \\
\text { a unit }\end{array}$ \\
\hline 1 & Filling & 26.77 & 80.31 & 0.75 & 1 & 0.75 & 26.77 & 26.77 \\
\hline 2 & $\begin{array}{l}\text { Capping and placing of } \\
\text { heat shrink film }\end{array}$ & 7.92 & 23.76 & 2.53 & 1 & 2.53 & 7.92 & 26.77 \\
\hline 3 & CR stacking & 16 & 6 & 10 & & 10 & 2 & \\
\hline 4 & Thermosealing & 4.95 & 14.86 & 4.04 & & 4.04 & 4.95 & \\
\hline 5 & $\begin{array}{l}\text { Coding and placement in } \\
\text { basket }\end{array}$ & 4.99 & 14.96 & 4.01 & 1 & 4.01 & 4.99 & 26.77 \\
\hline 6 & $\begin{array}{l}\text { Transport of basket to } \\
\text { conveyor belt and back }\end{array}$ & 10.72 & 10.72 & 5.6 & & 5.6 & 3.57 & \\
\hline
\end{tabular}

Source: Prepared by the authors. 
balancing analysis indicates that efficiency could increase to $95 \%$ if 2 operators were employed.

\subsection{Time study for vanilla $L$ product offering}

The $L$ whipping process involved 4 operators and 6 activities, which are: filling, capping, placing of heat shrink film, thermosealing, coding, placement in basket, and placement on conveyor belt. Time study for vanilla $L$ product offering is showed in Table 7.

In Table 8, it is determined that the bottleneck activity in vanilla $L$ product offering is filling, with a standard time of $5.42 \mathrm{~s} /$ unit (liter). In Table 9, a line efficiency of $63 \%$ is observed with 4 operators on the line; however, line balancing analysis indicates

Table 3. Overview of line balancing for vanilla CR product offering.

\begin{tabular}{|c|c|c|c|c|}
\hline Bottleneck & 0.75 & baskets/min & & \\
\hline Hourly performance & 44.82 & baskets/h & & \\
\hline Process duration & 6.51 & $\mathrm{~h}$ & 23424.5455 & $\mathrm{~s}$ \\
\hline No. of operators & 3 & operators & & \\
\hline Productivity & 14.94 & baskets/h * operators & & \\
\hline Line efficiency & 63 & $\%$ & & \\
\hline Cost person-hours & 81.32 & thousands of pesos & & \\
\hline Cycle time & 26.77 & s/unit & & \\
\hline No. of recommended stations & 2.00 & & & \\
\hline Cycle time & 26.8 & s/unit & & \\
\hline No. of recommended stations & 2 & & & \\
\hline Potential efficiency & 94 & $\%$ & & \\
\hline
\end{tabular}

Source: Prepared by the authors.

Table 4. Time study record sheet for vanilla CTP product offering.

\begin{tabular}{|c|l|c|c|c|c|c|c|}
\hline $\begin{array}{c}\text { Order of } \\
\text { activity }\end{array}$ & Activity & $\begin{array}{c}\mathbf{V} \\
\text { (performance } \\
\text { rating factor) }\end{array}$ & $\begin{array}{c}\text { Number of units } \\
\text { hold during certain } \\
\text { period }\end{array}$ & $\begin{array}{c}\text { Observed } \\
\text { time } \\
(\mathbf{s})\end{array}$ & $\begin{array}{c}\text { Basic or } \\
\text { Normal } \\
\text { time } \\
(\mathbf{s})\end{array}$ & $\begin{array}{c}\text { Allowance } \\
\text { time } \\
(\%)\end{array}$ & $\begin{array}{c}\text { Standard } \\
\text { time } \\
(\mathbf{s})\end{array}$ \\
\hline 1 & Filling & 1 & 1 & 21.02 & 21.02 & 1.00 & 21.02 \\
\hline 2 & Capping & 1.02 & 1 & 3.95 & 4.03 & 1.07 & 4.31 \\
\hline 3 & CTP stacking & 0.98 & 9 & 49.47 & 48.48 & 1.05 & 5.66 \\
\hline 4 & Placement in basket & 0.98 & 1 & 4.82 & 4.72 & 1.03 & 4.86 \\
\hline 5 & $\begin{array}{l}\text { Transport of basket to } \\
\text { conveyor belt and back }\end{array}$ & 0.96 & 3 & 10.53 & 10.11 & 1.06 & 10.72 \\
\hline
\end{tabular}

Source: Prepared by the authors.

Table 5. Line balancing analysis for vanilla CTP product offering.

\begin{tabular}{|c|l|l|c|c|c|c|c|c|}
\hline $\begin{array}{c}\text { Order of } \\
\text { activity }\end{array}$ & Activity & $\begin{array}{l}\text { Standard } \\
\text { time } \\
\text { (s/unit) }\end{array}$ & $\begin{array}{c}\text { Standard } \\
\text { time } \\
\text { (s/3 units) }\end{array}$ & $\begin{array}{c}\text { Baskets * } \\
\text { operatorl } \\
\text { min }\end{array}$ & $\begin{array}{c}\text { No. of } \\
\text { operators }\end{array}$ & $\begin{array}{c}\text { Total units } \\
\text { (No. of baskets/ } \\
\text { min) }\end{array}$ & $\begin{array}{c}\text { Time it takes } \\
\text { an operator } \\
\text { to produce a } \\
\text { unit }\end{array}$ & $\begin{array}{c}\text { Maximum } \\
\text { time used to } \\
\text { produce a } \\
\text { unit }\end{array}$ \\
\hline 1 & Filling & 21.02 & 63.07 & 0.95 & 1 & 0.95 & 21.02 & 21.02 \\
\hline 2 & Capping & 4.31 & 12.94 & 4.64 & 1 & 4.64 & 4.31 & 21.02 \\
\hline 3 & CTP stacking & 50.90 & 16.97 & 3.54 & 1 & 3.54 & 6.36 & 21.02 \\
\hline 4 & $\begin{array}{l}\text { Placement in } \\
\text { baskets }\end{array}$ & 4.86 & 14.59 & 4.11 & & 4.11 & 4.86 & \\
\hline 5 & $\begin{array}{l}\text { Transport of } \\
\text { basket to conveyor } \\
\text { belt and back }\end{array}$ & 10.72 & 10.72 & 5.60 & & 5.60 & 3.57 & \\
\hline & & & & & 3 & & 40.13 & 63.07 \\
\hline
\end{tabular}

Source: Prepared by the authors. 
that efficiency could increase to $84 \%$ if 3 operators were employed.

\section{Proposed strategies to improve whipping efficiency}

Using the data obtained, the following strategies are proposed to improve the efficiency of the whipping process. As for the filling activity for the CR, CTP and $L$ product offering, time can be reduced with the acquisition of a more efficient freezer or by enhancing the freezer's freezing capacity, which in turn would increase filling speed. It is also recommended to carry out the line balancing of the studied product offerings, which account for $80 \%$ of sales, in order to have an impact on its production efficiency.

In addition, two ice cream freezers with the same capacity should be assembled by means of a single pipe in order to reduce the filling time by half, employing almost the same number of operators, which would represent an improvement in efficiency. Finally, it is suggested to redistribute the complementary equipment and tools used in the whipping process through a study of methods, in order to reduce the number of operators and increase production line efficiency.

\section{CONCLUSIONS}

The standard time for vanilla CR, CTP and L product offerings was 50.2 s/unit, 40.1 s/unit and 12.7 s/unit, respectively. In this distribution, the bottleneck for all product offerings analyzed was the filling activity.

Current efficiency for CR, CTP and L product offerings is $63 \%, 64 \%$ and $63 \%$, respectively, and potential efficiency for line balancing said product offerings is $94 \%, 95 \%$ and $85 \%$, respectively.

Table 6. Overview of line balancing for vanilla CTP product offering.

\begin{tabular}{|l|c|c|c|c|}
\hline Bottleneck & 0.95 & baskets/min & & \\
\hline Hourly performance & 57.08 & baskets/h & & \\
\hline Process duration & 5.84 & $\mathrm{~h}$ & 21021.82 & $\mathrm{~s}$ \\
\hline No. of operators & 3 & operators & & \\
\hline Productivity & 19.03 & baskets/h operator & & \\
\hline Line efficiency & 64 & \% & & \\
\hline Cost person hours & 72.98 & thousands of pesos & & \\
\hline Cycle time & 21.02 & s/unit & & \\
\hline No. of recommended stations & 2 & & & \\
\hline Cycle time & 21.02 & s/unit & & \\
\hline No. of recommended stations & 2 & & & \\
\hline Potential efficiency & 95 & $\%$ & & \\
\hline
\end{tabular}

Source: Prepared by the authors.

Table 7. Time study record sheet for vanilla $L$ product offering.

\begin{tabular}{|l|l|c|c|c|c|c|c|}
\hline $\begin{array}{l}\text { Order of } \\
\text { activity }\end{array}$ & Activity & $\begin{array}{c}\mathbf{V} \\
\text { (performance } \\
\text { rating factor) }\end{array}$ & $\begin{array}{c}\text { Number of } \\
\text { units hold } \\
\text { during certain } \\
\text { period }\end{array}$ & $\begin{array}{c}\text { Observed } \\
\text { time } \\
\mathbf{( s )}\end{array}$ & $\begin{array}{c}\text { Basic or Nor- } \\
\text { mal time } \\
\mathbf{( s )}\end{array}$ & $\begin{array}{c}\text { Allowance } \\
\text { time } \\
\mathbf{( \% )}\end{array}$ & $\begin{array}{c}\text { Standard } \\
\text { time } \\
(\mathbf{s})\end{array}$ \\
\hline 1 & Filling & 1 & 1 & 5.42 & 5.42 & 1.00 & 5.42 \\
\hline 2 & Capping & 1.04 & 1 & 2.23 & 2.32 & 1.07 & 2.48 \\
\hline 3 & Placing of shrink heat film & 1.02 & 1 & 1.29 & 1.31 & 1.05 & 1.38 \\
\hline 4 & Thermosealing & 1.02 & 1 & 1.86 & 1.90 & 1.03 & 1.95 \\
\hline 5 & Coding & 0.98 & 1 & 0.80 & 0.78 & 1.03 & 0.80 \\
\hline 6 & $\begin{array}{l}\text { Transport of basket to } \\
\text { conveyor belt and back }\end{array}$ & 0.97 & 15 & 23.54 & 22.83 & 1.06 & 24.20 \\
\hline
\end{tabular}

Source: Prepared by the authors. 
Tabla 8. Line balancing analysis for vanilla $L$ product offering.

\begin{tabular}{|c|l|c|c|c|c|c|c|c|}
\hline $\begin{array}{c}\text { Order of } \\
\text { activity }\end{array}$ & Activity & $\begin{array}{c}\text { Standard } \\
\text { time } \\
\text { (s/unit) }\end{array}$ & $\begin{array}{c}\text { Standard } \\
\text { time } \\
\mathbf{( s / 1 5} \\
\text { units) }\end{array}$ & $\begin{array}{c}\text { Baskets * } \\
\text { operator/min }\end{array}$ & $\begin{array}{c}\text { No. of op- } \\
\text { erators }\end{array}$ & $\begin{array}{c}\text { Total units } \\
\text { (No. of bas- } \\
\text { kets/min) }\end{array}$ & $\begin{array}{c}\text { Time it takes } \\
\text { an operator } \\
\text { to produce a } \\
\text { unit }\end{array}$ & $\begin{array}{c}\text { Maximum } \\
\text { time used to } \\
\text { produce a } \\
\text { unit }\end{array}$ \\
\hline 1 & Filling & 5.42 & 81.37 & 0.74 & 1 & 0.74 & 5.42 & 5.42 \\
\hline 2 & Capping & 2.48 & 37.17 & 1.61 & 1 & 1.61 & 2.48 & 5.42 \\
\hline 3 & $\begin{array}{l}\text { Placing of shrink } \\
\text { heat film }\end{array}$ & 1.38 & 20.69 & 2.90 & & 2.90 & 1.38 & \\
\hline 4 & Thermosealing & 1.95 & 29.30 & 2.05 & 1 & 2.05 & 1.95 & 5.42 \\
\hline 5 & Coding & 0.80 & 12.06 & 4.98 & 1 & 4.98 & 0.80 & 5.42 \\
\hline 6 & $\begin{array}{l}\text { transport of basket } \\
\text { to conveyor belt and } \\
\text { back }\end{array}$ & 24.20 & 24.20 & 2.48 & & 2.48 & 1.61 & \\
\hline
\end{tabular}

Source: Prepared by the authors.

Tabla 9. Overview of line balancing for vanilla $L$ product offering.

\begin{tabular}{|l|c|c|c|c|}
\hline Bottleneck & 0.74 & baskets/min & & \\
\hline Hourly performance & 44.24 & baskets/h & & \\
\hline Process duration & 5.65 & $\mathrm{~h}$ & 20342.308 & $\mathrm{~s}$ \\
\hline No. of operators & 4 & operators & & \\
\hline Productivity & 11.06 & baskets/h * operator & & \\
\hline Line efficiency & 63 & $\%$ & & \\
\hline Cost person hours & 94.16 & thousands of pesos & & \\
\hline Cycle time & 5.42 & s/unit & & \\
\hline No. of recommended stations & 3 & & & \\
\hline Cycle time & 5.42 & s/unit (liter) & & \\
\hline No. of recommended stations & 3 & & & \\
\hline Potential efficiency & 84 & $\%$ & & \\
\hline
\end{tabular}

Fuente: Elaboración propia.

The number of operators working at present in the whipping process of the vanilla CR, CTP and $L$ product offerings is 3,3 and 4, respectively; the number proposed in the production line balancing is 2, 2 and 3 respectively.

\section{REFERENCES}

[1] Albi, E. (1992). Evaluación de la eficiencia pública. (El control de eficiencia del sector público). Hacienda Pública Española, 120-121, 299-316.

[2] Alfonso, A. \& Tamayo, Á. F. (2016). Diseño del sistema de gestión de procesos y operaciones para la administración del mantenimiento industrial en Helados Popsy. (Master thesis). Universidad Sergio Arboleda, Bogotá.

[3] Caso, A. (2006). Sistema de incentivos a la producción. Madrid, Spain: FC Editorial.
[4] Chase R., Jacobs R. \& Aquilano N. (2009). Administración de operaciones. Producción y cadena de suministros. México D. F., Mexico: Mc Graw Hill.

[5] Cruelles, J. A. (2013). Productividad e incentivos. Cómo hacer que los tiempos de fabricación se cumplan. México D. F., Mexico: Alfaomega / Marcombo.

[6] García, R. (2005). Estudio del trabajo. Ingeniería de métodos y medición del trabajo. México D. F., Mexico: McGraw-Hill.

[7] González, L. A. \& Jácome, A. H. (2012). Elaboración de una propuesta de mejora para el proceso productivo del helado de crema de una empresa manufacturera en la ciudad de Guayaquil. (Undergraduate thesis). Escuela Superior Politécnica del Litoral, Guayaquil. 
[8] Heizer, J. \& Render, B. (2009). Principios de administración de operaciones. México D. F., Mexico: Pearson Educación.

[9] Hodson, W. K. (2009). Maynard. Manual del Ingeniero Industrial. México D. F., México: McGraw-Hill.

[10] Kanawaty, G. (1996). Introducción al estudio del trabajo. Ginebra, Suiza: Oficina Internacional del Trabajo.

[11] Meyers, F. E. (2000). Estudios de tiempos y movimientos. Para la manufactura ágil. México D. F., Mexico: Pearson Educación

[12] Meyers, F. E. \& Stephens, M. P. (2006). Diseño de instalaciones de manufactura y manejo de materiales. México D. F., Mexico: Pearson Educación.
[13] Muñoz, D. J. (2006). Estandarización de los procesos de producción de los productos elaborados para los puntos de venta de Yogen Früz. (Undergraduate thesis). Universidad de La Salle, Bogotá.

[14] Rodríguez, J. (2008). Determinación del tiempo estándar para la actualización de las ayudas visuales en una línea de producción de una empresa manufacturera. (Undergraduate thesis). Instituto Tecnológico de Sonora, Sonora.

[15] Salazar, B. (June 26, 2019). Valoración del ritmo de trabajo. [Blog entry]. Ingeniería Online. Retrieved from https://www. ingenieriaindustrialonline.com/estudio-detiempos/valoracion-del-ritmo-de-trabajo. 